\title{
The Dehn twist on a sum of two K3 surfaces
}

\author{
P. B. Kronheimer and T. S. Mrowka \\ Harvard University, Cambridge MA 02138 \\ Massachusetts Institute of Technology, Cambridge MA 02139
}

\begin{abstract}
Ruberman gave the first examples of self-diffeomorphisms of four-manifolds that are isotopic to the identity in the topological category but not smoothly so. We give another example of this phenomenon, using the Dehn twist along a 3-sphere in the connected sum of two $K 3$ surfaces.
\end{abstract}

\section{Introduction}

A 2-dimensional Dehn twist is a non-trivial self-diffeomorphism of an annulus, fixing the two boundary circles pointwise. As a generalization of this, for any $n \geq 2$, there is a self-diffeomorphism of the $n$-manifold $[0,1] \times S^{n-1}$,

$$
\delta_{n}:[0,1] \times S^{n-1} \rightarrow[0,1] \times S^{n-1}
$$

having the form $\delta_{n}(t, s)=\left(t, \alpha_{t}(s)\right)$, where $\alpha:[0,1] \rightarrow S O(n)$ is a loop based at the identity element lying in the non-trivial homotopy class. We can arrange that $\delta_{n}$ is the identity near both boundary components, and this allows to extend $\delta_{n}$ to a diffeomorphism of any $n$-manifold $X$ provided only that an embedding of $[0,1] \times S^{n-1}$ in $X$ is given. The resulting diffeomorphism $\delta: X \rightarrow X$ is referred to as a Dehn twist along the sphere $S^{n-1} \subset[0,1] \times S^{n-1}$. In particular if $X$ is a connected sum $X_{1} \# X_{2}$, then one can consider the Dehn twist along the separating sphere in the neck. In this paper, we shall prove:

Theorem 1.1. Let $Z$ be the connected sum K3\# K3, and let $\delta: Z \rightarrow Z$ be a Dehn twist along the separating $S^{3}$ in the neck. Then the diffeomorphism $\delta$ is not isotopic to the identity.

The work of the first author was supported by the National Science Foundation through NSF grant DMS-1707924. The work of the second author was supported by NSF grant DMS-1808794. 
It is evident that $\delta$ induces the identity map on homology, and by a theorem of Quinn [12], applicable to closed simply-connected four-manifolds in general, it follows that $\delta$ lies in the identity component of the homeomorphism group $\operatorname{Top}(Z)$. Theorem 1.1 implies that it does not lie in the identity component of $\operatorname{Diff}(Z)$. The first example of this phenomenon - an element in the kernel of the map $\pi_{0}(\operatorname{DifF}(X)) \rightarrow \pi_{0}(\operatorname{Top}(X))$ for a smooth 4-manifold $X$ - was given by Ruberman in [13]. Additional examples were presented later by Baraglia and Konno in [2]. The theorem above provides the first example where the Dehn twist on a connected sum of simply connected 4-manifolds has been shown to be non-trivial.

The techniques we employ here are drawn from the same toolkit that has been used to detect other non-smoothability results for families in dimension four: gauge theory (here the Seiberg-Witten equations), the numerical invariants of families that they can be used to define $[13,14,9]$, and the homotopy refinements of these that one may construct in the spirit of [6] and [16].

The next statement, which is a corollary of the theorem above, was proved earlier by Baraglia and Konno in [4]. (The version here is not explicitly stated in [4], but an equivalent reformulation, Proposition 2.1 below, is a special case of their results, as we explain in the next section.)

Proposition 1.2 (cf. [4]). Let $X^{\prime}$ be the 4-manifold with boundary obtained by removing an open ball from a K3 surface. Let $\delta: X^{\prime} \rightarrow X^{\prime}$ be the diffeomorphism supported in the interior of $X^{\prime}$ obtained by a Dehn twist along a 3-sphere parallel to the boundary. Then $\delta$ is not isotopic to the identity element in the group $\operatorname{DiFF}\left(X^{\prime}, \partial X^{\prime}\right)$ of diffeomorphisms which fix the boundary pointwise.

In this paper, we will reprove Proposition 1.2, using Bauer-Furuta invariants for families in a slightly different way, and we will deduce Theorem 1.1 using a product theorem for connect sums modeled on [5].

Acknowledgements. Conversations with Sander Kupers and Danny Ruberman were very helpful for the formulation of these results and in the preparation of this paper. In particular, the authors would like to thank Sander Kupers for pointing out the relevance of [10] in the higher-dimensional case.

\section{Families of spin manifolds}

To explain the connection between Proposition 1.2 and the results of [4], consider first the action of $\operatorname{Diff}(K 3)$ on the frame bundle $F(K 3)$ and the resulting map 
$e: \operatorname{DifF}(K 3) \rightarrow F(K 3)$ which one obtains by applying this to a basepoint $\theta$ in $F(K 3)$. The map $e$ is a fibration whose fiber (the stabilizer of $\theta$ ) has the weak homotopy type of the group $\operatorname{DifF}\left(X^{\prime}, \partial X^{\prime}\right)$ and the exact sequence of the fibration gives

$$
\pi_{1}(\operatorname{DiFF}(K 3)) \stackrel{e_{*}}{\longrightarrow} \pi_{1}(F(K 3)) \longrightarrow \pi_{0}\left(\operatorname{DiFF}\left(X^{\prime}, \partial X^{\prime}\right)\right.
$$

The fundamental group of $F(K 3)$ is $\mathbb{Z} / 2$ (as it is for any simply-connected spin manifold), and the class of the Dehn twist $\delta$ in $\pi_{0}\left(\operatorname{DifF}\left(X^{\prime}, \partial X^{\prime}\right)\right)$ is the image of the generator of $\pi_{1}(F(K 3))$. The assertion in Corollary 1.2, that $\delta$ is non-trivial, is therefore equivalent to saying that the map

$$
e_{*}: \pi_{1}(\operatorname{DifF}(K 3)) \rightarrow \mathbb{Z} / 2
$$

is zero.

The map $e_{*}$ can be interpreted in yet another way. Given a loop $\gamma$ in $\operatorname{DifF}(K 3)$, form the fiber bundle $E \rightarrow S^{2}$ with fiber $K 3$, using $\gamma$ as the clutching function. Then $e_{*}[\gamma]$ is equal to the evaluation of $w_{2}(T E)$ on any section of $E$. An elementary restatement of the vanishing of $e_{*}$ is therefore the following:

Proposition 2.1 ([4]). Let $E \rightarrow S^{2}$ be a smooth fiber bundle, with fiber a K3 surface. Then the Stiefel-Whitney class $w_{2}(T E)$ is zero.

The above proposition is a consequence of [4, Corollary 1.3] and the arguments of [4, Section 4.2]. As shown in [3], the corresponding statement in the topological category is false: there is a non-smoothable topological family of $K 3$ surfaces over $S^{2}$ which has non-trivial Stiefel-Whitney class.

There is a third reformulation of Propositions 1.2 and 2.1, which is the one most convenient for our discussion of the Bauer-Furuta invariants below. To set up the general context, let $X$ be a smooth, oriented, closed 4-manifold, which we assume at present is connected and simply connected with even intersection form. In the absence of a Riemannian metric, a "spin structure" on $X$ can be defined as a lift of the structure group of the oriented frame bundle of $X$, from $S L(4, \mathbb{R})$ to the double cover $\tilde{S L}(4, \mathbb{R})$. Our hypotheses imply that $X$ admits a spin structure $\mathfrak{s}$, which is unique up to isomorphism. The group of automorphisms of $\mathfrak{s}$ is the group of order 2 generated by the deck transformation of the double cover.

We write $\operatorname{SDifF}(X)$ for the group of orientation-preserving diffeomorphisms of $X$. The group $\operatorname{SDiff}(X)$ has a double cover $\operatorname{DiffSpin}(X) \rightarrow \operatorname{SDiff}(X)$ consisting of pairs $(f, i)$, where $f: X \rightarrow X$ is a diffeomorphism and $i: f^{*}(\mathfrak{s}) \rightarrow \mathfrak{s}$ is 
an isomorphism of spin structures. Given an element $h \in \operatorname{DifFSPIN}(X)$, we can form a mapping torus $X^{h}$ fibering over the circle $B=[0,1] / \sim$, together with a spin structure $\mathfrak{s}^{h}$ on the vertical tangent bundle of the fibration.

As a special case we can consider the element $\tau=(1, t)$ in $\operatorname{DiffSpin}(X)$, where 1 denotes the identity on $X$ and $t$ denotes the deck transformation of the spin structure. We shall prove,

Proposition 2.2 ([4]). If $X$ is a K3 surface, then the involution $\tau$ in $\operatorname{DifFSpin}(X)$ is not in the identity component. Equivalently, the family of spin manifolds $\left(X^{\tau}, \mathfrak{s}^{\tau}\right)$ over the circle $B$ is not isomorphic to the trivial family $B \times(X, \mathfrak{s})$.

It follows from the discussion that this proposition is an equivalent reformulation of both Propositions 1.2 and 2.1. Indeed, the double cover $\operatorname{DiffSPIN}(X) \rightarrow$ $\operatorname{SDiff}(X)$ is classified by a map $\pi_{1}(\operatorname{SDifF}(X)) \rightarrow \mathbb{Z} / 2$ which is easily identified with the map $e_{*}$ in (1). Proposition 2.2 says that this double cover is trivial for $K 3$, which is equivalent therefore to the vanishing of $e$. We will prove Proposition 2.2 in section 4, after introducing the tools from Seiberg-Witten theory in section 3.

\section{Bauer-Furuta invariants for spin families}

To fix our conventions and context, we summarize in this section the techniques of finite-dimensional approximation, as applied to the Seiberg-Witten equations on 4-manifolds, first for a single 4-manifold as in [7, 6], and then for families of 4-manifolds over a base, as developed and explored first in [16], and later in $[15,1,3]$, for example. We focus on the case that $X$ is equipped with a spin structure, rather than a more general $\operatorname{spin}^{c}$ structure, and we assume that $b_{1}(X)=$ 0 .

So let $X$ be a closed, oriented 4-manifold with $b_{1}=0$. Let $\mathfrak{s}$ be a spin structure on $X$. After equipping with the manifold with a Riemannian metric, the spin structure gives rise to spin bundles $S^{+}, S^{-}$over $X$, Clifford multiplication $\gamma$ : $\Lambda^{1} \otimes S^{+} \rightarrow S^{-}$, and the Dirac operator $D: \Gamma\left(S^{+}\right) \rightarrow \Gamma\left(S^{-}\right)$. The Seiberg-Witten map is a non-linear Fredholm map between Hilbert spaces,

$$
S W: \mathscr{W}^{+} \rightarrow \mathscr{W}^{-}
$$

Unwrapping this a bit, we have

$$
\mathscr{W}^{+}=\mathscr{V}^{+} \oplus \mathcal{U}^{+}, \quad \mathscr{W}^{-}=\mathscr{V}^{-} \oplus \mathcal{U}^{-},
$$


where $\mathscr{V}^{ \pm}$are suitable Sobolev completions of $\Gamma\left(S^{ \pm}\right)$, and $\mathcal{U}^{+}, \mathcal{U}^{-}$are Sobolev completions of respectively $\Omega^{1}(X)$ and $\Omega^{+}(X) \oplus \Omega^{0}(X) / \mathbb{R}$. The Seiberg-Witten map has the form $S W=l+c$, where $l$ is the Fredholm operator

$$
l=D \oplus\left(d^{+}, d^{*}\right)
$$

and $c$ has the form

$$
c(a, \phi)=(\gamma(i a, \phi), q(\phi, \bar{\phi}))
$$

where $q$ is a bilinear term.

Now let $W^{-} \subset \mathscr{W}^{-}$be a finite-dimensional subspace, large enough that $W^{-}+\operatorname{im}(l)=\mathscr{W}^{-}$. Set $W^{+}=l^{-1}\left(W^{-}\right)$. The corresponding finite-dimensional approximation to the Seiberg-Witten map is constructed in [6] as the map

$$
s w=(\rho \circ S W): W^{+} \rightarrow W^{-},
$$

where $\rho: \mathscr{W}^{-} \backslash S\left(\left(W^{-}\right)^{\perp}\right) \rightarrow W^{-}$is a suitable retraction. It is shown in [6] that if $W^{-}$is sufficiently large, then the image of $S W$ does not intersect the unit sphere $S\left(\left(W^{-}\right)^{\perp}\right)$ in the orthogonal complement, so the composite $\rho \circ S W$ is indeed defined. The finite-dimensional approximation is a proper map and extends to the one-point compactifications as a map of spheres:

$$
[s w] \in\left[W_{\infty}^{+}, W_{\infty}^{-}\right]
$$

We will take $W^{-}$always of the form $V^{-} \oplus U^{-}$, in which case also $W^{+}=$ $V^{+} \oplus U^{+}$. Furthermore, $\mathscr{V}^{ \pm}$are quaternion vector spaces and $D$ is quaternionlinear, and we are therefore able to insist also that $V^{ \pm}$are quaternion vector subspaces.

The operator $\left(d^{+}+d^{*}\right)$ is injective with cokernel $H^{+}(X)$, the space of harmonic self-dual 2-forms. We may choose $U^{-}$to contain this space, so that

$$
U^{-}=u^{-} \oplus H^{+}
$$

and $l: U^{+} \rightarrow u^{-}$is a linear isomorphism. A choice of orientation of $H^{+}$then allows us to identify the orientation lines of $U^{+}$and $U^{-}$. The vector spaces $V^{ \pm}$ are naturally oriented themselves, because they are quaternion vector spaces. In all then, the orientation of $\mathrm{H}^{+}$allows us to identify the orientation lines of $W^{ \pm}$.

Let $M$ be a regular fiber of the finite-dimensional approximation $s w$, over a point in $p \in W^{-}$. As the fiber of map between relatively oriented vector spaces, $M$ is naturally a stably framed manifold. The results of [6] imply that, provided 
$W^{-}$is sufficiently large, the fiber $M$ is compact, and its framed cobordism class depends only on $X$ and the orientation of $\mathrm{H}^{+}$, not on the choice of Riemannian metric or on the choice of $W^{-}$. Here "sufficiently large" means only that $W^{-}$ should contain a subspace $W_{*}^{-}(g)$ which depends on the metric $g$. The dimension of $M$ is

$$
d=-\frac{1}{4}(2 e+3 \sigma)+1
$$

where $e$ and $\sigma$ are the Euler number and signature of $X$.

Framed cobordism classes of $d$-manifolds are classified by the stable $d$-stem $\pi_{d}^{s}=\pi_{d+N}\left(S^{N}\right)$ for $N$ large. Instead of referring to the regular fiber of $s w$, we can refer directly to the homotopy class of the map $s w$ itself, as a map between spheres as in (2). For the purposes of this paper, we use the following strippeddown version of the Bauer-Furuta invariant:

Definition 3.1. The Bauer-Furuta invariant of the spin manifold $(X, \mathfrak{s})$, with the chosen orientation of $H^{+}$, is the framed cobordism class $\eta(X)$ of the regular fiber of $s w$, for any metric $g$ and any choice of $W^{-}$containing $W_{*}^{-}(g)$.

Now suppose instead of a single 4-manifold we have a smooth fiber bundle $X \rightarrow B$ over a compact base. Let $\mathfrak{s}$ be a given fiberwise spin structure, and let a family of Riemannian metrics be given. The spaces $\mathscr{W}^{+}$and $\mathscr{W}^{-}$are now bundles over $B$, and $S W$ is a bundle map. After choosing a suitable finite-rank subbundle $W^{-} \subset \mathscr{W}^{-}$, we have finite-dimensional approximations $s w: W^{+} \rightarrow$ $W^{-}$, where $W^{+}=V^{+} \oplus U^{+}$and $W^{-}=V^{-} \oplus U^{-}$are finite-rank vector bundles over $B$. This construction is defined whenever $W^{-}$is sufficiently large, which can be taken to mean that $W^{-}$contains a certain subbundle $W_{*}^{-}$depending on the metric. The map $s w$ is proper and therefore extends to the fiberwise one-point compactifications: it becomes a map of based sphere bundles, with a homotopy class

$$
[s w] \in\left[W_{\infty}^{+}, W_{\infty}^{-}\right] .
$$

Let $s$ be a smooth section of $W^{-} \rightarrow B$, transverse to $s w$. The inverse image $s w^{-1}(s)$ is then a compact manifold $M$ with a map to $B$. The dimension of $M$ is $\operatorname{dim}(B)+d$, where $d$ is the invariant of the 4 -dimensional fiber, as above.

To go further, we impose extra conditions to ensure that we can stably trivialize the bundles $W^{ \pm}$canonically. First, the bundles $V^{ \pm}$are quaternionic, and $\operatorname{Sp}(N)$ is 2-connected. So if we require that $\operatorname{dim} B \leq 2$, then these bundles have preferred trivializations. To trivialize $U^{+} \ominus U^{-}$stably is again equivalent to trivializing $H^{+}$viewed now as a bundle over $B$. There is an action of $\pi_{1}(B)$ on the 
homology $H^{2}\left(X_{b}\right)$ of the fiber $X_{b}$. If we impose the condition that this action is trivial, then $H^{+}$is a maximal positive-definite subbundle of the trivial vector bundle $H^{2}\left(X_{b} ; \mathbb{R}\right)$, and it therefore has a preferred trivialization as $B \times H^{+}\left(X_{b}\right)$ because the space of maximal positive-definite subspaces of $H^{2}\left(X_{b} ; \mathbb{R}\right)$ is a contractible subset of the Grassmannian. An orientation of $\mathrm{H}^{+}$for any fiber therefore completely determines a trivialization.

At this point, the map $p: M \rightarrow B$ has a relative stable framing: a stable trivialization of $T M \ominus T B$. It is convenient in the exposition to make $M$ itself stably framed, and to do so we ask that $B$ have a stable framing of its tangent bundle. We are specifically interested in the case that $B$ is the circle equipped with the stable framing which bounds the framed disk. Recall from the introduction, that if $(X, \mathfrak{s})$ is a spin 4-manifold and $h \in \operatorname{DifFSPIN}(X, \mathfrak{s})$, then we can construct a spin family $\left(X^{j}, \mathfrak{s}^{h}\right)$ over the base $B=S^{1}$ as the mapping torus of $h$. The Bauer-Furuta construction now produces a framed manifold $M\left(X^{h}, \mathfrak{s}^{h}\right)$. We summarize this as follows.

Definition 3.2. Let $(X, \mathfrak{s})$ be a closed, oriented spin 4-manifold with $b_{1}=0$ and let $h \in \operatorname{DifFSpin}(X, \mathfrak{s})$ be an element which acts trivially on $H_{2}(X)$. Equip the circle $B$ with the bounding stable framing and let $M$ be the resulting framed manifold of dimension $1+d$, defined as $M=s w^{-1}(s)$, for a generic section $s$ of $W^{-}$. The Bauer-Furuta invariant $\eta\left(X^{h}, \mathfrak{s}^{h}\right)$ of $h$ is the framed cobordism class of $M$, or equivalently the corresponding element of $\pi_{d+1}^{s}$.

Our choice to give $B$ the framing which bounds means that the invariant $\eta$ is zero for the trivial product family over $B$. So if $\eta\left(X^{h}, \mathfrak{s}^{h}\right)$ is non-zero, then $h$ is not in the identity component of $\operatorname{DifFSPIN}(X, \mathfrak{s})$.

Remark. As the discussion makes clear, the construction in this form applies equally well if $B$ is, for example, a 2-sphere. Thus, if $(X, \mathfrak{s})$ is a spin manifold with $b_{1}=0$, then the construction provides a homomorphism $\pi_{1}(\operatorname{DiffSPIN}(X, \mathfrak{s})) \rightarrow$ $\pi_{d+2}^{s}$.

\section{Calculation for the twisted $K 3$ family}

Let $X$ again be a closed, oriented 4-manifold with $b_{1}=0$, equipped with a spin structure $\mathfrak{s}$. Let $\tau \in \operatorname{DifFSpIN}(X, \mathfrak{s})$ be the deck transformation of $\mathfrak{s}$, the generator of the kernel of $\operatorname{DiffSpin}(X, \mathfrak{s}) \rightarrow \operatorname{SDiff}(X)$. We can form the spin family $\left(X^{\tau}, \mathfrak{s}^{\tau}\right)$ over $B$, and the invariant $\eta\left(X^{\tau}, \mathfrak{s}^{\tau}\right)$. 
Proposition 4.1. If the signature of $X$ is equal to $16 \bmod 32$ (i.e. if the complex index of D is $2 \bmod 4$ ), then the Bauer-Furuta invariant of the twisted spin family $\left(X^{\tau}, \mathfrak{s}^{\tau}\right)$ over the circle $B$ is given by a product

$$
\eta\left(X^{\tau}, \mathfrak{s}^{\tau}\right)=\eta_{1} \times \eta(X, \mathfrak{s})
$$

as a cobordism class of stably framed $(d+1)$-manifolds. Here $\eta_{1}$ is the non-trivial element of $\pi_{1}^{s}$, represented by the circle with Lie-group framing, and $\eta(X, \mathfrak{s})$ is the Bauer-Furuta invariant of $(X, \mathfrak{s})$, as a stably framed d-manifold. If the signature of $X$ is equal to 0 mod 32 , then $\eta\left(X^{\tau}, \mathfrak{s}^{\tau}\right)$ is zero.

Corollary 4.2. If $X$ is a K3 surface, then $\eta\left(X^{\tau}, \mathfrak{s}^{\tau}\right)$ is non-zero.

Proof of the Corollary. The Bauer-Furuta invariant of $K 3$ with its unique spin structure $\mathfrak{s}$ is the class $\eta_{1}$, represented by the Lie-framed circle. The signature of $K 3$ is 16 , so the proposition above tells us that the invariant of the family $\left(X^{\tau}, \mathfrak{s}^{\tau}\right)$ is $\eta_{1} \times \eta_{1}$. This is the generator of the stable 2 -stem, $\pi_{2}^{s}=\mathbb{Z} / 2$.

Proposition 2.2 follows directly from this result, as do the reformulations, Propositions 1.2 and 2.1 .

Proof of Proposition 4.1. Let

$$
s w: W^{+} \rightarrow W^{-}
$$

be the finite-dimensional approximation of the Seiberg-Witten map for the spin manifold $X$ itself, equipped with some metric $g$. As usual we write $W^{+}=V^{+} \oplus U^{+}$ and similarly with $W^{-}$. The vector spaces $V^{ \pm}$are quaternion vector spaces, and we adopt the convention that the quaternion scalars $I, J, K$ act on the left. The circle group acts on $V^{+}$and $V^{-}$by left-multiplication by $e^{I \theta}$. We extend this action to all of $W^{ \pm}$by making the action trivial on $U^{ \pm}$. The Seiberg-Witten map commutes with this circle action, as does its finite-dimensional approximation.

Over the interval $[0,1]$, form the trivial product bundles $[0,1] \times W^{ \pm}$. For any $\theta \in[0, \pi]$, let $W_{\theta}^{+} \rightarrow B$ be the vector bundle obtained by identifying $\{1\} \times W^{+}$ with $\{0\} \times W^{+}$using left-multiplication by $e^{I \theta}$ :

$$
\{1\} \times W^{+} \stackrel{e^{I \theta} \times}{\longrightarrow}\{0\} \times W^{+} .
$$

Define $W_{\theta}^{-} \rightarrow B$ similarly. 
The map $s w$ commutes with $e^{I \theta}$, so it gives rise to a bundle map over $B$, for each $\theta$,

$$
s w_{\theta}: W_{\theta}^{+} \rightarrow W_{\theta}^{-} .
$$

When $\theta=0$, this is (the finite-dimensional approximation to) the Seiberg-Witten map for the trivial family $B \times(X, \mathfrak{s})$ over the circle. When $\theta=\pi$ this is the SeibergWitten map for the twisted family $\left(X^{\tau}, \mathfrak{s}^{\tau}\right)$, because the involution $\tau$ acts as -1 on the spin bundles $S^{ \pm}$.

We now wish to compare the two proper bundle maps

$$
\begin{aligned}
& s w_{0}: W_{0}^{+} \rightarrow W_{0}^{-} \\
& s w_{\pi}: W_{\pi}^{+} \rightarrow W_{\pi}^{-} .
\end{aligned}
$$

On the one hand we have a proper isotopy between them, given by the bundle maps

$$
s w_{\theta}: W_{\theta}^{+} \rightarrow W_{\theta}^{-},
$$

for $\theta \in[0, \pi]$. However, multipliction on the left by $e^{I \theta}$ is not a quaternion-linear transformation for intermediate values $\theta \in(0, \pi)$, so we do not have an isotopy through a family of quaternion vector bundles $V_{\theta}^{ \pm}$. The trivializations of $V_{\pi}^{ \pm}$arising from their quaternionic structure may be different then the trivializations they aquire from the trivial bundles $V_{0}^{ \pm}$via this isotopy.

To compare the trivializations, we construct a different isotopy over $[0, \pi] \times B$ between the vector bundles $V_{0}^{ \pm}$and $V_{\pi}^{ \pm}$. To do so we trivialize the fiber $V^{+}$of the trivial bundle $V_{0}^{+}$as $\mathbb{H}^{n}$. We construct a vector bundle $\hat{W}_{\theta}^{+}$over $B$, for $\theta \in[0, \pi]$, in just the same was as we defined $W_{\theta}^{+}$before, but now using right-multiplication by $e^{I \theta}$ on $\mathbb{\boxplus}^{n}$ instead of left-multiplication. We do the same with $\hat{W}_{\theta}^{-}$. The SeibergWitten map does not commute with this action, so does not define a bundle map; but we are concerned only with the trivializations. Because right-multiplication is quaternion linear, we now have vector bundles $\hat{W}_{\theta}^{ \pm}$, providing an isotopy over $[0, \pi] \times B$ between $W_{0}^{ \pm}$and $W_{\theta}^{ \pm}$, and the " $V$ " summands of these are quaternion vector bundles. The trivialization of $W_{\theta}^{ \pm}$that we are required to use is the one that arises from the trivial bundle via this new isotopy.

To compare the trivializations that arise via these two different isotopies, consider composing the first with the second. On the $\mathbb{T}^{n}$ summand corresponding to $V^{+}$, we have an isotopy over $[0,2 \pi] \times B$ from the trivial bundle over $B$ with fiber $\mathbb{R}^{4 n}$ back to itself. The total bundle over $[0,2 \pi] \times B$ is constructed from the trivial bundle over $[0,2 \pi] \times[0,1]$ by identifying the fibers using a certain path $[0,2 \pi] \rightarrow S O(4 n)$. This path is the concatenation of a first path from 1 to -1 
given by left multiplication by $e^{I \theta}$ with a second path from -1 to 1 given by right multiplication by $e^{I \theta}$. In the case $n=1$, such a path from 1 to 1 belongs to the non-trivial homotopy class in $\pi_{1}(S O(4))$. In general it is non-trivial in $\pi_{1}(S O(4 n))$ if and only if $n$ is odd.

The same arguments apply to $W^{-}$as well, so when we consider a relative framing of $W_{\pi}^{+} \ominus W_{\pi}^{-}$, we see that the stable framing acquired from the trivial bundle by the isotopy (3) is equal to the quaternionic framing if

$$
\operatorname{dim}_{\mathbb{\sharp}} V^{+}-\operatorname{dim}_{\llbracket} V^{-}
$$

is even. Otherwise, the framings differ by the non-trivial map $B \rightarrow S O$. (This difference is half the complex index of $D$.)

In terms of the stably framed manifold $M_{\tau}$ representing the Bauer-Furuta invariant of the twisted family $\left(X^{\tau}, \mathfrak{s}^{\tau}\right)$, the conclusion is that $M_{\tau}$ is framedcobordant to the product $B \times M$ (and therefore to $\varnothing$ ) if the index of $D$ is $0 \mathrm{mod}$ 4 , and is framed-cobordant to $L \times M$ if the index is $2 \bmod 4$, where $L$ is the circle with non-zero framing.

\section{Connected sums}

Let $\left(X, \mathfrak{s}_{X}\right)$ and $\left(Y, \mathfrak{s}_{Y}\right)$ be two spin 4-manifolds. We suppose that both have $b_{1}=0$ so that our exposition of the Bauer-Furuta invariants applies. Remove standard balls from each and identify collars of the boundary 3-spheres by a diffeomorphism $\psi$ so as to form the oriented connected sum $X \#_{0} Y$. We write $C_{X}$ and $C_{Y}$ for these (closed) collars and

$$
\psi: C_{X} \rightarrow C_{Y}
$$

for the diffeomorphism. Let $\alpha:[0,1] \rightarrow S O(4)$ be a closed loop in the non-trivial homotopy class. For each $t \in[0,1]$ we can form a connected sum $X \#_{t} Y$, using $\psi \circ \alpha(t)$ in place of $\psi$. The 4-manifolds $X \#_{0} Y$ and $X \#_{1} Y$ are identical, so we may form a family of 4-manifolds over the circle $B=[0,1] /$, which we write as

$$
p: X \#_{\alpha} Y \rightarrow B
$$

The derivative of $\psi$ identifies the frame bundles of the two collars. Lift this to an isomorphism $\tilde{\psi}$ of the spin bundles $\left.\left.\mathfrak{s}_{X}\right|_{C_{X}} \rightarrow \mathfrak{s}_{Y}\right|_{C_{Y}}$. There are two such lifts: $\tilde{\psi}$ and $\tilde{\psi} \circ \tau$, where $\tau$ is the deck transformation: choose one of them. Thought of as a path in $\operatorname{DifF}\left(C_{X}\right)$, there is a unique lifting of $\alpha$ to a path $\tilde{\alpha}$ in $\operatorname{DifF} \operatorname{SpIN}\left(C_{X}, \mathfrak{s}_{X}\right)$ 
starting at the identity. Because the homotopy class of $\alpha$ in $S O(4)$ is non-trivial, its lift is not a closed loop and we have $\tilde{\alpha}(1)=\tau$. So if we equip $B \times X$ with the twisted family of spin structures $\mathfrak{s}^{\tau}$ and equip $B \times Y$ with the product family, then we can form a spin family

$$
\left(X^{\tau} \#_{\alpha} Y, \mathfrak{s}_{X}^{\tau} \#_{\tilde{\alpha}} \mathfrak{s} Y\right) .
$$

We could also do the twist $\tau$ on the side of $Y$ rather than $X$.

Proposition 5.1. For the family of 4-manifolds (4) over the circle B with fiber $X \#_{0} Y$, equipped with the family of spin structures (5), the Bauer-Furuta invariant $\eta$ is given by

$$
\eta_{1} \times \eta\left(X, \mathfrak{s}_{X}\right) \times \eta\left(Y, \mathfrak{s}_{Y}\right)
$$

if $\sigma(X)$ is $16 \bmod 32$, and zero otherwise.

Remark. There is an asymmetry in the conclusion here (only the signature of $X$ matters, not the signature of $Y$ ) because of the asymmetry in the construction of the family of spin structures.

Proof. Let us abbreviate our notation a little by taking the spin structures as implied. We write $X^{\tau}$ for the family over $B$ with twisted spin structure, and we write $X^{\tau} \#_{\alpha} Y$ for the result of summing $Y$ to each fiber of $X^{\tau}$ using the path $\alpha$ to vary the gluing. The assertion of the proposition can then be rephrased (using Proposition 4.1) as:

$$
\eta\left(X^{\tau} \#_{\alpha} Y\right)=\eta\left(X^{\tau}\right) \times \eta(Y)
$$

This is the product formula for the Bauer-Furuta invariants from [5], extended to the case of families.

The proof from [5] extends without much difficulty. To clarify this, we repeat the main setup steps from [5], in the families context. Let $Z^{a} \rightarrow B$ be a finite collection of closed 4-manifolds over a base $B$, indexed by $a \in A$, a finite set. Let $Z$ be the disjoint union. For each $a$, suppose there is a decomposition of $Z^{a}$ as a fiberwise connected sum, realized explicitly by a smooth embedding of a family of collars $C^{a}$ over $B$. That is, $C^{a} \subset Z^{a}$ is a bundle over $B$ with fiber $[-1,1] \times S^{3}$, which we take to be equipped with a family of metrics isometric to the standard one on each fiber. Let $C \hookrightarrow Z$ be the union. We can write

$$
Z=Z_{+} \cup Z_{-}, \quad C=Z_{+} \cap Z_{-}
$$

where $Z_{+}$and $Z_{-}$are families of manifolds with boundary, with boundary components indexed by $A$. Let $\sigma: C \rightarrow C$ be an automorphism of $C$ over the base 
$B$, which permutes the components by an even permutation of $A$. Let $Z^{\sigma}$ be obtained as the union of $Z_{+}$and $Z_{-}$, attached along $C$ using the automorphism $\sigma$.

If $Z$ is given a fiberwise spin structure, and if $\sigma: C \rightarrow C$ is lifted to an isomorphism of spin families, then $Z^{\sigma}$ also acquires the structure of a spin family over $B$. We have Bauer-Furuta invariants arising from finite-dimensional approximations $s w$ and $s w^{\sigma}$ for these two families. (For a disjoint union, the Seiberg-Witten map $S W$ is defined to be the fiber product over $B$.)

In the case that $B$ is a point, the construction of $Z^{\sigma}$ from $Z$ is the same setup as in [5], and in Section 3 of that paper as series of homotopies is constructued, to show that that finite-dimensional approximations $s w$ and $s w^{\sigma}$ are homotopic. The same homotopies can be applied fiberwise over $B$, because all the estimates can be made uniformly over the compact base. This establishes that $s w$ and $s w^{\sigma}$ are properly homotopic bundle maps over $B$. As in [5], the application to a connected sum of two manifolds is deduced by considering the case that $|A|=3$ and taking the fibers of $Z$ to be

$$
\left(X \# S^{4}\right) \sqcup\left(S^{4} \# Y\right) \sqcup\left(S^{4} \# S^{4}\right) .
$$

A cyclic permutation $\sigma$ of order 3 is even and the resulting family $Z^{\sigma}$ has fibers

$$
(X \# Y) \sqcup\left(S^{4} \# S^{4}\right) \sqcup\left(S^{4} \# S^{4}\right) .
$$

A family of 4-spheres over the circle has two possible spin structures, related by the twist $\tau$, but the resulting two spin families are isomorphic. The BauerFuruta invariant of either family is represented by the identity map between zerodimensional vector bundles over $B$, which contributes trivially. So the homotopy between $s w$ and $s w^{\sigma}$ identifies the invariant of the family with fibers $X \# Y$ with that of the family with fibers $X \sqcup Y$.

We now return to the first theorem stated in this paper.

Proof of Theorem 1.1. Consider the case that $X$ and $Y$ are both $K 3$ surfaces, and form the family of 4-manifolds

$$
p: X \#_{\alpha} X \rightarrow B, \quad(X=K 3) .
$$

There is a unique spin structure up to isomorphism on the fiber, and there are two ways to equip the family over $B$ with fiberwise spin structures. In the notation of the constructions above, these are the spin families $X^{\tau} \#_{\alpha} X$ and $X \#_{\alpha} X^{\tau}$. 
The previous proposition says that the Bauer-Furuta invariant for each of these families is $\left(\eta_{1}\right)^{3}$, because $\eta(X)=\eta_{1}$. The cube of $\eta_{1}$ is the element of order 2 in $\pi_{3}^{s}=\mathbb{Z} / 24$, and in particular is non-zero. It follows that the underlying family of smooth 4-manifolds $X \#_{\alpha} X$ is a non-trivial family over the circle. The monodromy of this family is the mapping class of the Dehn twist $\delta$ supported in the collar where the connected sum consruction is made. It follows that $\delta$ is not isotopic to the identity.

\section{Additional remarks}

More Dehn twists. The non-triviality of $\delta$ in Proposition 1.2 is a question raised in a more general form by Giansiracusa in [8]. To describe this, let $X$ be a simplyconnected, closed, spin 4-manifold, and let $X^{(n)}$ be obtained from $X$ by removing $n$ disjoint balls. Let $\operatorname{DifF}\left(X^{(n)}, \partial\right)$ denote the group of diffeomorphisms which are the identity in a neighborhood of the boundary, so that there is map

$$
\operatorname{DifF}\left(X^{(n)}, \partial\right) \rightarrow \operatorname{DifF}\left(X^{(n)}\right) .
$$

It is shown in [8] that the corresponding map on $\pi_{0}$ has kernel equal to either $(\mathbb{Z} / 2)^{n-1}$ or $(\mathbb{Z} / 2)^{n}$. The ambiguity results from the question of whether the particular diffeomorphism $\delta^{(n)}$, defined as the composite of the Dehn twists around the $n$ disjoint spheres parallel to the boundary components, is isotopic to the identity in $\operatorname{DifF}\left(X^{(n)}, \partial\right)$. For given $X$, the answer to this question is independent of $n$. Proposition 1.2 is the statement that $\delta^{(n)}$ is non-trivial in the case of a K3 surface. As pointed out in [8], the isotopy class of $\delta^{(n)}$ is trivial if $w_{2}(X)$ is nonzero, and is also trivial in the case of $S^{2} \times S^{2}$ as one can see by using a circle action on the manifold.

Homotopy $K 3$ surfaces. It follows from the results of [11] and [6] that $\eta(X)=$ $\eta_{1}$ for any homotopy $K 3$ surface $X$. Theorem 1.1 therefore applies equally well to homotopy K3 surfaces.

Exploiting equivariance of $s w$. The Seiberg-Witten maps $s w$ are equivariant for the action of the group $\operatorname{Pin}(2) \subset S p(1)$ generated by the circle $e^{I \theta}$ and the element $J$. These act by multiplication on the quaternion vector spaces $V^{ \pm}$. On $U^{ \pm}$the circle action is trivial and $J$ acts by -1 . This extra structure is exploited in [7] and in [6,5], but we have not used it here except in an auxiliary role, to construct an isotopy in the proof of Proposition 4.1. It would be interesting to 
see if the equivariant version can be used to extend Theorem 1.1 to some other cases.

Higher dimensions. In higher dimensions, the possible non-triviality of the boundary Dehn twist in Proposition 1.2 is a question addressed in detail by Kreck in [10] for almost-parallelizable $(k-1)$-connected $2 k$-manifolds; and in the same setting, the results there allow one to determine when a Dehn twist on the neck of a connected sum is non-trivial. Specifically, let $X$ be a $(k-1)$-connected and almost parallelizable $2 k$-manifold, and let $X^{\prime}$ denote the manifold-with-boundary obtained by removing a ball. Then in the notation of [10] there is assigned to $X$ an element $\Sigma_{X}$ of order at most two in the group $\Theta_{2 k+1}$ of homotopy spheres of dimension 1 higher. This assignment is additive for connected sums, and if the dimension of $X$ is at least 6, then $\Sigma_{X}$ is zero if and only if the Dehn twist on the sphere parallel to the boundary of $X^{\prime}$ is zero in $\pi_{0}\left(\operatorname{DifF}\left(X^{\prime}, \partial X^{\prime}\right)\right)$. For a connected sum $X_{1} \# X_{2}$, it can also be deduced from [10] that the Dehn twist around the neck is trivial if and only if $\Sigma_{X_{1}}$ is zero in the quotient $\Theta_{2 k+1} /\left(\Sigma_{X_{1} \# X_{2}}\right)$. Since these elements have order at most 2, this criterion for non-triviality is simply that $\Sigma_{X_{1}}$ and $\Sigma_{X_{2}}$ are both non-zero.

As an example of the computations in [10], if the dimension is 8, then $\Sigma_{X}$ is non-zero if the index of the Dirac operator on $X$ is odd. If the index of the Dirac operator on $X$ is even, then the vanishing of $\Sigma_{X}$ is dependent on the smooth structure. As a special case, if $X$ is the exotic sphere of dimension 8 , then the Dehn twist in the neck of $X \# X$ is a non-trivial element of $\pi_{0}(\operatorname{DifF}(X \# X))$. In this case, the connected sum $X \# X$ is $S^{8}$ and it is presented as the union of two balls: the non-triviality of the Dehn twist arises from the non-standard parametrization of the standard 7-sphere along which the Dehn twist is performed.

\section{References}

[1] David Baraglia. Constraints on families of smooth 4-manifolds from Bauer-Furuta invariants. Preprint, 2019.

[2] David Baraglia and Hokuto Konno. A gluing formula for families Seiberg-Witten invariants. Preprint, 2018.

[3] David Baraglia and Hokuto Konno. A note on the Nielsen realization problem for K3 surfaces. Preprint, 2019. 
[4] David Baraglia and Hokuto Konno. On the Bauer-Furuta and Seiberg-Witten invariants of families of 4-manifolds. Preprint, 2019.

[5] Stefan Bauer. A stable cohomotopy refinement of Seiberg-Witten invariants. II. Invent. Math., 155(1):21-40, 2004.

[6] Stefan Bauer and Mikio Furuta. A stable cohomotopy refinement of Seiberg-Witten invariants. I. Invent. Math., 155(1):1-19, 2004.

[7] M. Furuta. Monopole equation and the $\frac{11}{8}$-conjecture. Math. Res. Lett., 8(3):279-291, 2001.

[8] Jeffrey Giansiracusa. The stable mapping class group of simply connected 4manifolds. F. Reine Angew. Math., 617:215-235, 2008.

[9] Tsuyoshi Kato, Hokuto Konno, and Nobuhiro Nakamura. Rigidity of the mod 2 families Seiberg-Witten invariants and topology of families of spin 4-manifolds. Preprint, 2019.

[10] M. Kreck. Isotopy classes of diffeomorphisms of $(k-1)$-connected almostparallelizable $2 k$-manifolds. In Algebraic topology, Aarhus 1978 (Proc. Sympos., Univ. Aarhus, Aarhus, 1978), volume 763 of Lecture Notes in Math., pages 643-663. Springer, Berlin, 1979.

[11] John W. Morgan and Zoltán Szabó. Homotopy K3 surfaces and mod 2 SeibergWitten invariants. Math. Res. Lett., 4(1):17-21, 1997.

[12] Frank Quinn. Isotopy of 4-manifolds. F. Differential Geom., 24(3):343-372, 1986.

[13] Daniel Ruberman. An obstruction to smooth isotopy in dimension 4. Math. Res. Lett., 5(6):743-758, 1998.

[14] Daniel Ruberman. A polynomial invariant of diffeomorphisms of 4-manifolds. In Proceedings of the Kirbyfest (Berkeley, CA, 1998), volume 2 of Geom. Topol. Monogr., pages 473-488. Geom. Topol. Publ., Coventry, 1999.

[15] Markus Szymik. Characteristic cohomotopy classes for families of 4-manifolds. Forum Math., 22(3):509-523, 2010.

[16] Ming Xu. The Bauer-Furuta invariant and a cohomotopy refined Ruberman invariant. ProQuest LLC, Ann Arbor, MI, 2004. Thesis (Ph.D.)-State University of New York at Stony Brook. 